Supporting Information For

\title{
Crystal Structures of L-DOPA Dioxygenase from Streptomyces sclerotialus
}

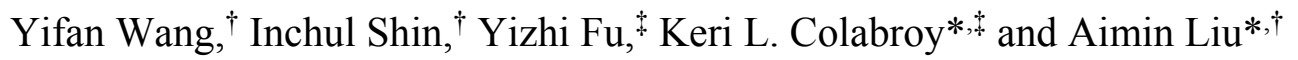

${ }^{\dagger}$ Department of Chemistry, University of Texas at San Antonio, San Antonio, TX 78249; and

Department of Chemistry, Muhlenberg College, Allentown, PA, United States

\section{Correspondence}

*Aimin Liu: E-mail: Feradical@utsa.edu; Tel: +1-210-458-7062

* Keri L. Colabroy: E-mail: colabroy@muhlenberg.edu; Tel: +1-484-664-3665 
Table S1. Summary of ligand distances $(\AA)$ to iron center in each of the six subunits (A-F). Asterisks indicate residues from another subunit.

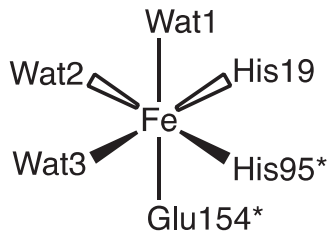

Resting state structure

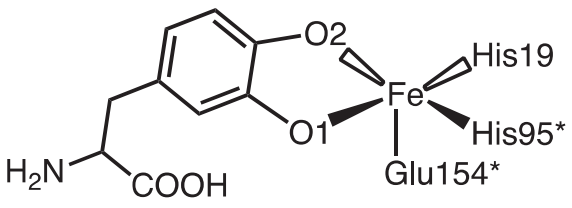

Substrate bound structure

\begin{tabular}{cccccccc}
\hline \multicolumn{2}{c}{ Distance } & Subunit A & Subunit B & Subunit C & Subunit D & Subunit E & Subunit F \\
\hline $\begin{array}{c}\text { Resting } \\
\text { state } \\
\text { structure }\end{array}$ & Wat1 & N/A & 2.11 & 2.09 & 2.00 & 2.00 & 2.06 \\
\hline $\begin{array}{c}\text { Substrate } \\
\text { bound }\end{array}$ & Wat3 & N/A & 2.26 & 2.27 & 2.28 & 2.30 & 2.49 \\
structure & $\mathrm{O} 2$ & 2.53 & 2.53 & 2.47 & 2.53 & 2.65 & 2.49 \\
\hline
\end{tabular}

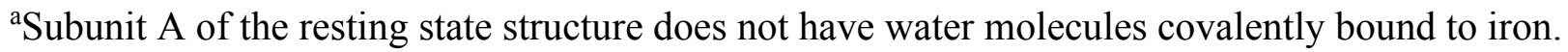




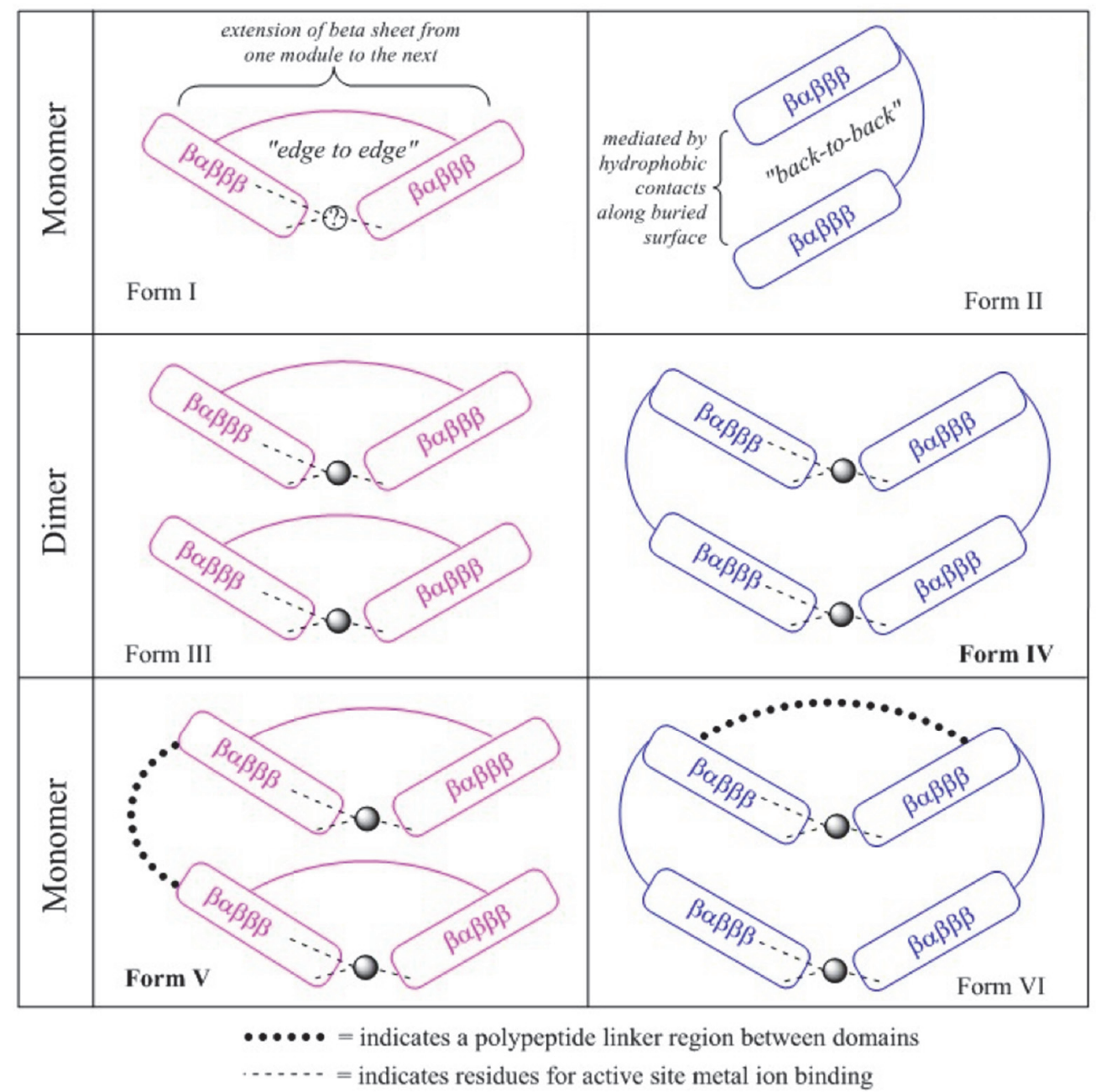

Figure S1. Six possible VOC superfamily topologies. VOC family proteins are assemblies of different $\beta \alpha \beta \beta \beta$ modules. If the VOC protein binds a metal, the metal chelating site (represented by a grey sphere) is formed by edge-to-edge contacts of two $\beta 1$ strands from different $\beta \alpha \beta \beta \beta$ modules. The functional class of non-redox VOC enzymes contains representative domain architecture of every possible Form (I though VI), with the exception of the monomeric Form II. Characterized VOC/Type I extradiol dioxygenase are almost exclusively of Form V architecture. The assignment of domain arrangements as "Forms I - VI" is from He and Moran (Structural and mechanistic comparisons of the metal-binding members of the vicinal oxygen chelate (VOC) superfamily, J. Inorg. Biochem. 2011, 105, 1259-1272). 


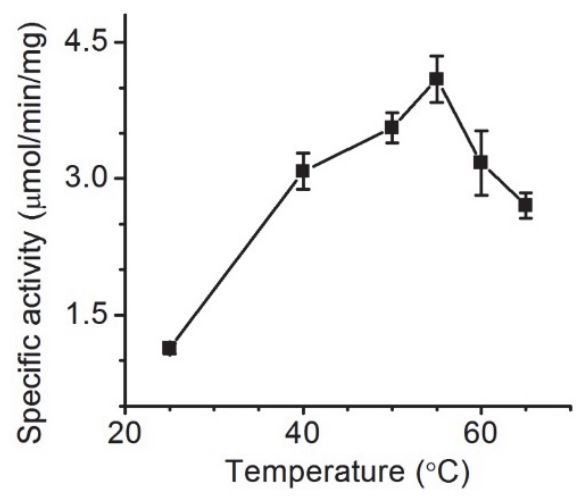

Figure S2. Temperature-dependent activity assay. SsDDO specific activity was measured with saturating L-DOPA $(1 \mathrm{mM})$ at various temperatures. Steady-state kinetic assay of SsDDO with L-DOPA at 25 and $55^{\circ} \mathrm{C}$, obtained using UV-vis spectrometer. 


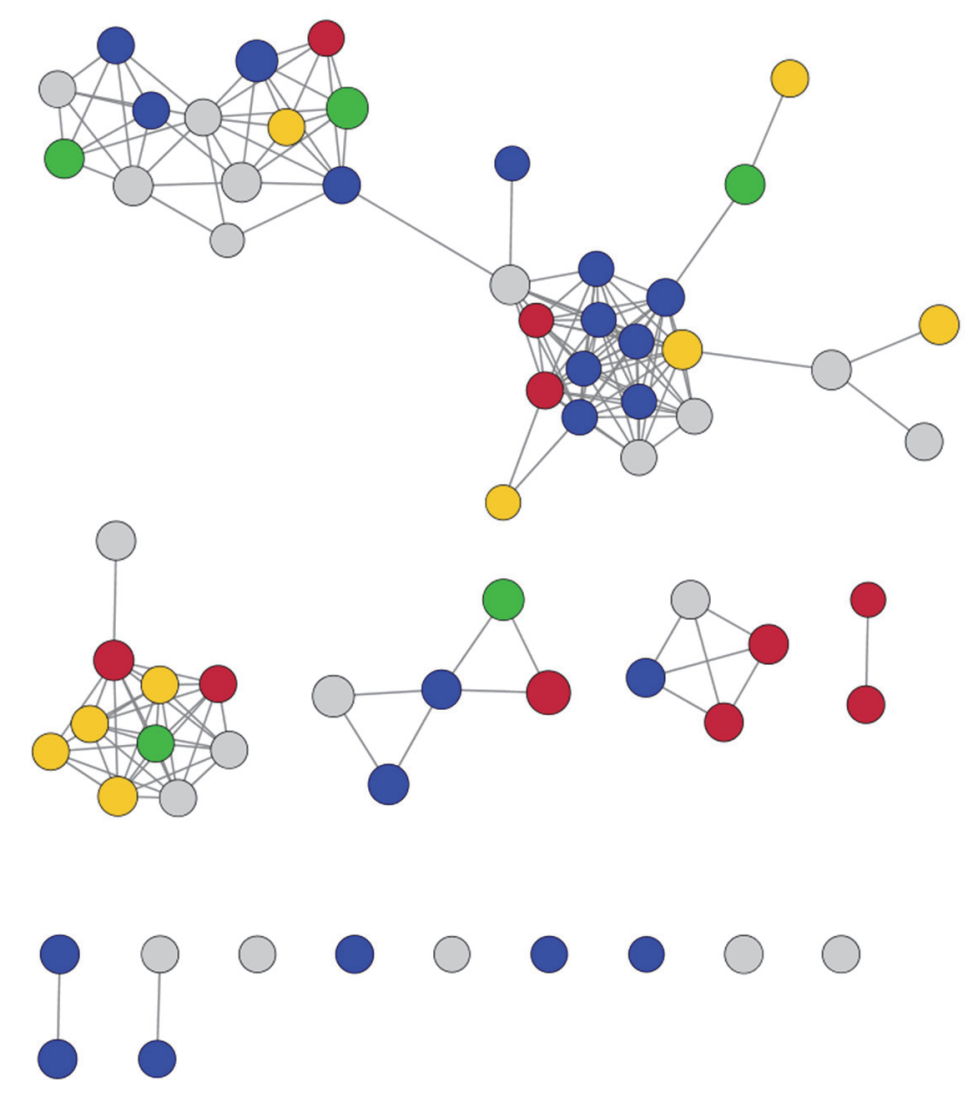

Figure S3. Sequence similarity network of SsDDO. Each node represents a protein sequence, the size of which is proportianal to the sequence length. Two nodes are connected by an edge when they share certain similarities. There are 65 nodes with 116 edges on that network ( $e$-value threshhold of $10^{-55}$ ). Proteins with different functions are in different colors: L-DOPA-2,3dioxygenases (red); catachol 2,3-dioxygense-type enzymes (yellow); dioxygenses (blue); glyoxalase I-type enzymes (green); uncharacterized (grey). 


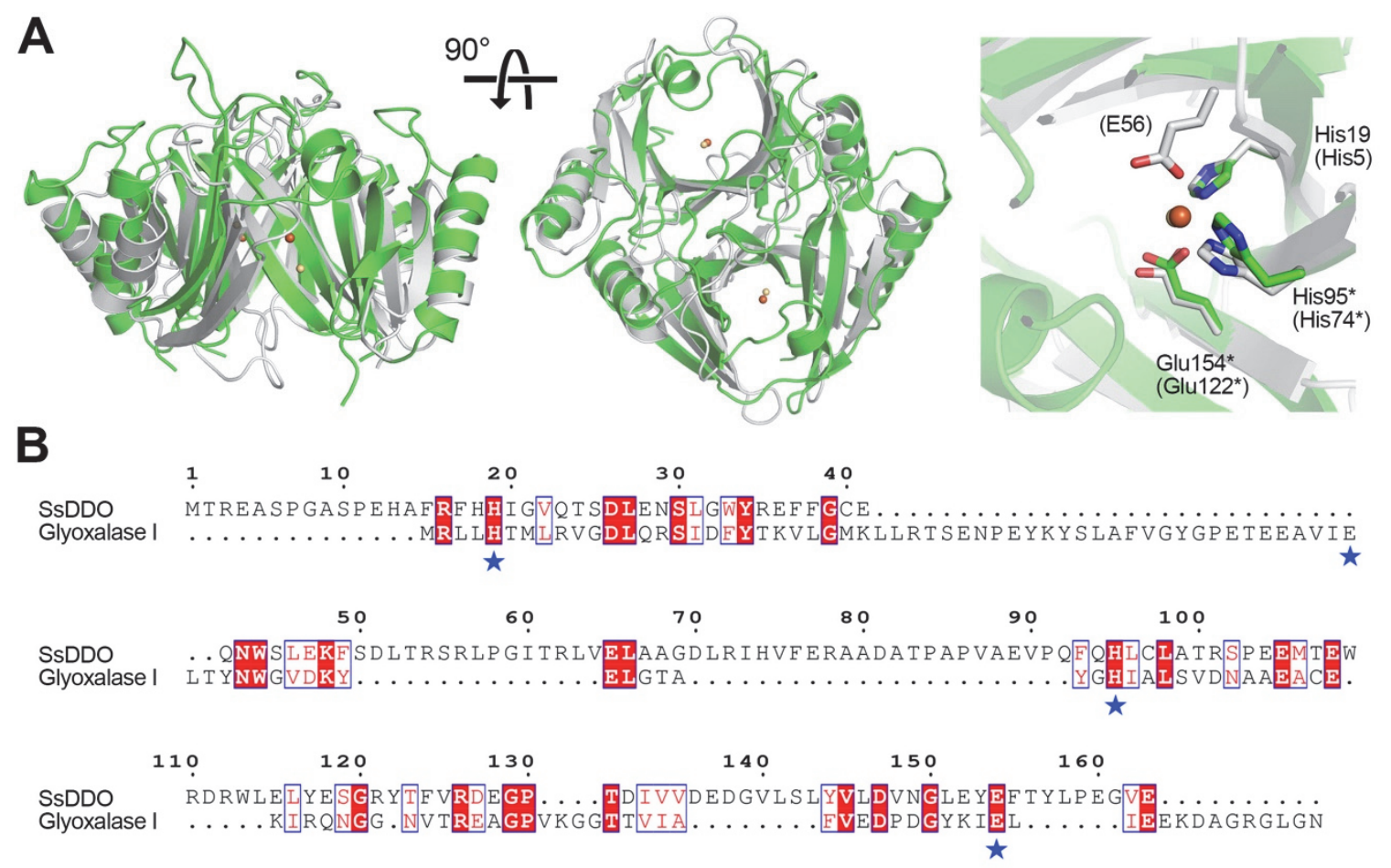

Figure S4. Comparison of SsDDO and glyoxalase I. (A) (Left) Superpose of global structures of SsDDO (green) and glyoxalase I (white, 1FA7). Orange and yellow spheres represent iron and cadmium, respectively. (Right) Active site view of SsDDO and glyoxalase I. The SsDDO amino acid residue numbers are followed by corresponding glyoxalase I residue numbers in parentheses. Asterisks indicate residues from another subunit. (B) Pairwise sequence alignment of SsDDO and glyoxalase I, a lactoylglutathione lyase (UniProtKB accession number: P0AC81). Highly conserved residues are shown in red. Strictly conserved residues are shown in white on a red background. Blue stars represent residues involved in the metal coordination. 


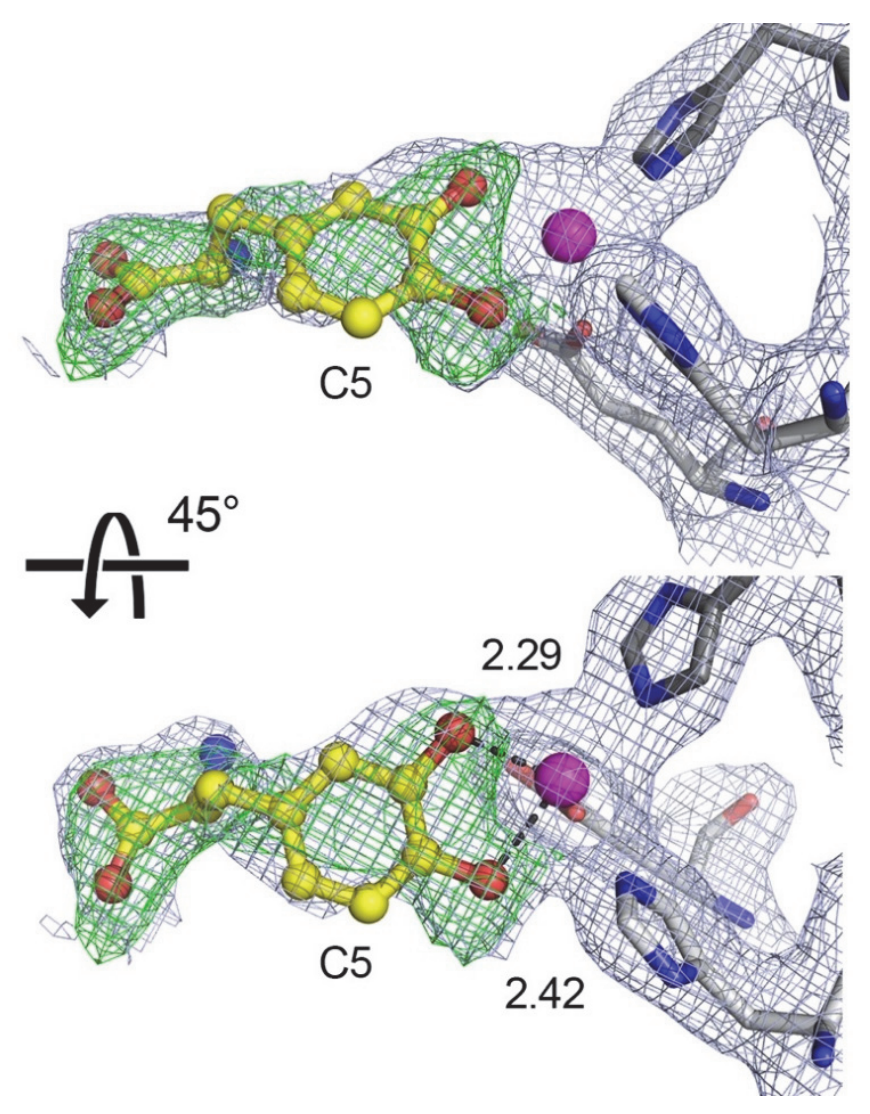

Figure S5. The fitting result with L-DOPA flipped aromatic ring. The density map missed partial electron density on C5 even though Fe-O1 was shorter than Fe-O2 (2.29/2.42 $\AA$ ). The green $F_{0^{-}}$ $F_{\mathrm{c}}$ omit map is contoured at $3 \sigma$ and the light blue $2 F_{\mathrm{o}}-F_{\mathrm{c}}$ electron density map after refinement is contoured at $1 \sigma$. Oxygen, nitrogen, iron atoms are represented in red, blue and magenta, respectively. Carbon atoms from subunit A, subunit B and L-DOPA are shown in white, dark and yellow. Distances are shown in dark dash lines. 


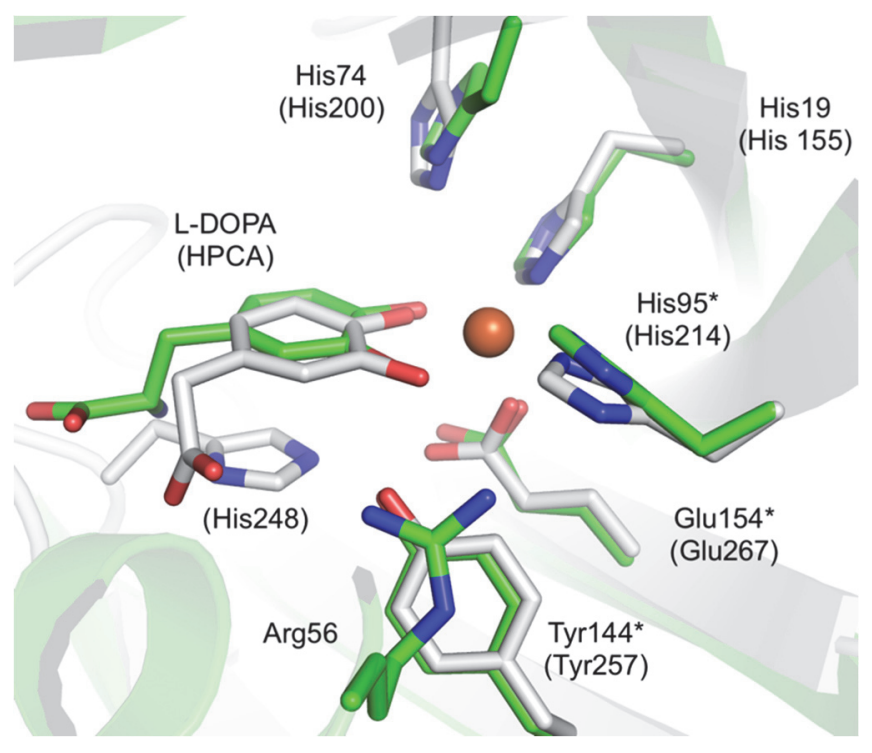

Figure S6. Superpose of the E.S complex structures of SsDDO and homoprotocatechuate 2,3dioxygenase (HPCD). SsDDO is complex with L-DOPA (green) and HPCD is complex with 2,3dihydroxyphenylacetate (HPCA) (white, PDB: 4GHG). SsDDO residue numbers are followed by corresponding HPCD residue numbers in parentheses. Asterisks indicate residues from another subunit. Oxygen, nitrogen, iron atoms are represented in red, blue and orange, respectively. 


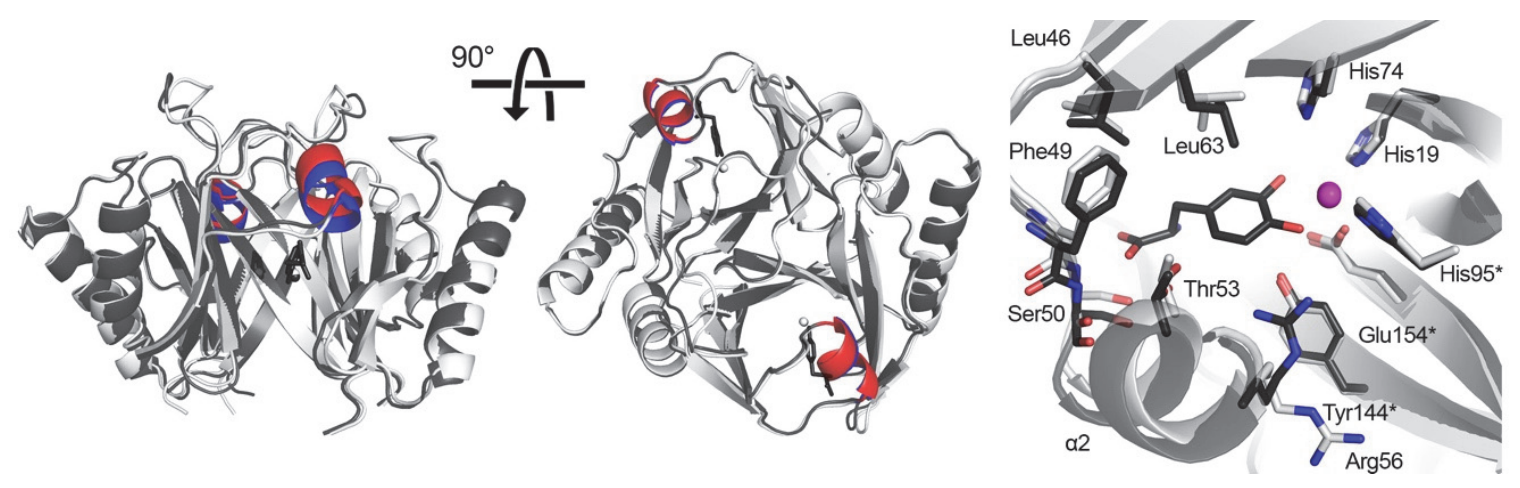

Figure S7. Global and zoom-in views of structural superpose of the resting state and binary E.S complex. The resting state structure is colored by white and blue, the E.S complex is colored black and red. A short a helix a2 (Asp51-Arg46) shows the greatest change before (blue) and after (red) L-DOPA binding. Oxygen, nitrogen, iron atoms are represented in red, blue and magenta, respectively. Residues with and without asterisks are from different subunits. 


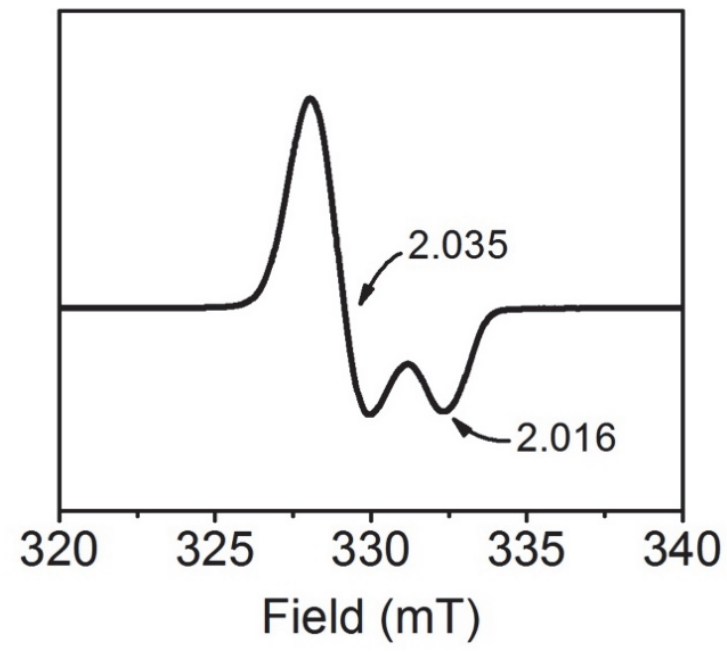

Figure S8. A dinitrosyl signal at $g=2$ region appeared in both E-NO• and E-S-NO• complexes. Substrate addition did not affect the linewidth and $g$ values of the dinitrosyl signal. Instrumental condition: microwave frequency $9.4 \mathrm{GHz}$, modulation frequency $100 \mathrm{kHz}$, modulation amplitude $6 \mathrm{G}$, temperature $50 \mathrm{~K}$, microwave power $0.05 \mathrm{~mW}$. 